\title{
Research on the Cutoff Tumor Size of Omitting Radiotherapy after Breast Conserving Therapy in Women Aged 65 Years or Oder with Low-risk Invasive Breast Carcinoma: Results Based on the SEER Database
}

\section{Zejian Yang}

Xi'an Jiaotong University Medical College First Affiliated Hospital

Kunlong Li

Xi'an Jiaotong University Medical College First Affiliated Hospital

\section{Bin Wang}

Xi'an Jiaotong University Medical College First Affiliated Hospital

Yu Yan

Xi'an Jiaotong University Medical College First Affiliated Hospital

\section{Chen Feng}

Xi'an Jiaotong University Medical College First Affiliated Hospital

\section{Yu Ren}

Xi'an Jiaotong University Medical College First Affiliated Hospital

\section{Du Meng}

Xi'an Jiaotong University Medical College First Affiliated Hospital

Yijun Li

Xi'an Jiaotong University Medical College First Affiliated Hospital

Yifei Ma

Xi'an Jiaotong University Medical College First Affiliated Hospital

Pei Qiu

Xi'an Jiaotong University

Pingping Li ( $\sim$ 13468845587@139.com )

Xi'an Jiaotong University Medical College First Affiliated Hospital

Can Zhou ( $\sim$ zhoucanz2005@xjtufh.edu.cn)

Xi'an Jiaotong University Medical College First Affiliated Hospital

\section{Research article}

Keywords: Breast-conserving surgery, Tumor size, Postoperative radiotherapy, SEER, X-tile analysis

Posted Date: August 19th, 2020

DOI: https://doi.org/10.21203/rs.3.rs-52133/v1

License: (1) This work is licensed under a Creative Commons Attribution 4.0 International License. Read Full License 


\section{Abstract \\ Background}

Radiotherapy (RT) after breast-conserving therapy (BCT) is not always necessary in older women staged T1N0M0 with low-risk invasive breast cancer, but few studies have concluded the detailed tumor size as a reference for avoiding RT. We aimed to explore and identify the cutoff tumor size for patients aged 65 or older with T1N0M0 stage and estrogen receptor positive (ER+), human epidermal growth factor receptor 2 positive (HER2-) breast cancer after BCT.

\section{Methods}

The study population was from the Surveillance, Epidemiology, and End Results (SEER) database in 2010 to 2016. Prognostic predictors associated with surgery were used by Kaplan-Meier analysis, X-tile analysis, competing risk model, Fine and Gray multivariable regression model, and propensity score matching (PSM) analysis.

\section{Results}

A total of 52049 women and 3846 deaths were included in the unmatched cohort, of which $11.93 \%$ (459/3846) were breast cancerspecific death (BCSD), and 88.07\% (3387/3846) were other cause-specific death (OCSD), with a median follow-up of 34 months. Based on the cutoff value of $14 \mathrm{~mm}$ determined by X-tile analysis, the study population were divided into small tumor group (tumor $\leq 14 \mathrm{~mm}$ in diameter) and large tumor group (tumor $\geq 15 \mathrm{~mm}$ in diameter). Subjects in small tumor group and large tumor with RT (large tumor \& RT) subgroup tended to have significantly lower cumulative BCSD (Gray's test, $P<0.001$ ) and OCSD (Gray's test, $P<0.001$ ) than those in large tumor with no RT (large tumor \& non-RT) subgroup, regardless of before or after PSM analysis. In competing risk regression mode analysis, patients in the large tumor group had worse prognosis in BCSD (HR: 2.091,95\% Cl 1.730-2.527, $P<0.001)$ than the small tumor group, even after the application of PSM (HR: 2.024, 95\% Cl 1.587-2.583, $P<0.001)$.

\section{Conclusion}

Our study demonstrated that small tumors (tumor $\leq 14 \mathrm{~mm}$ in diameter) were associated with better overall survival (OS), BCSD and OCSD, and radiotherapy might be omitted for patients aged 65 or older with T1NOM0 stage, ER + and HER2- small breast tumors after BCT.

\section{Background}

Adjuvant radiotherapy (RT) is a component of breast conserving therapy (BCT) in order to obtain locoregional control for patients with early stage breast cancer ${ }^{1-3}$. The administration of adjuvant RT could reduce both the risk of local relapse and 15 -year breast cancerspecific death (BCSD) irrespective of age ${ }^{1}$. However, advancing age was associated with more favorable tumor biology and elderly patients with breast cancer generally were considered having distinctive biologic and clinical characteristics ${ }^{4-7}$. And no significant advantage was gained in overall survival (OS) or disease-free survival from adjuvant RT after BCT by previous randomized controlled trials (CALGB 9343 and PRIME II), for elderly patients with T1-T2NOMO stage and estrogen receptor-positive (ER+) female breast cancer $(\mathrm{FBC})^{8,9}$. Such results indicated that postoperative RT after BCT is not always necessary in selected elderly women staged T1NOMO with $\mathrm{ER}+\mathrm{FBC}$. Moreover, as a relatively general term, stage T1 ranging from $1 \mathrm{~mm}$ to $20 \mathrm{~mm}$ in tumor size in the 7th edition of AJCC/TNM staging system for breast cancers, it was still unknown the detailed tumor size at which postoperative RT after BCT might be omitted without causing a significant reduction in both OS and breast cancer specific survival (BCSS).

A retrospective study based on SEER showed that for elderly women with hormone receptor-positive and T1N0 stage breast cancer, postoperative RT after BCT could be omitted only in patients who satisfy both a small tumor size $(\leq 10 \mathrm{~mm})$ and low tumor grade ${ }^{10}$. However, the study was simply grouped as 1-10 $\mathrm{mm}$ and 11-20 $\mathrm{mm}$ by tumor size. The detailed tumor size that define the cutoff point by which omission of RT could be considered for patients staged T1NOMO with low-risk (ER + and HER2-) invasive breast cancer remains uncertain. The age of 65 was commonly used as the threshold of old age ${ }^{11,12}$. For this, we have to renew the interest in identifying patients age 65 years or older with indolent tumors who are unlikely to die of their tumors and could avoid $\mathrm{RT}^{13}$. 
To further explore and identify the detailed tumor size which could affect the prognosis OS and BCSS for patients aged 65 years or more with negative lymph nodes and ER-positive,T1 stage FBC, we conducted a large cohort of women with FBC from 2010 to 2016 from the population-based database Surveillance, Epidemiology, and End Results (SEER) cancer registry program.

\section{Methods \\ Data Source}

We used data from the national cancer institute's SEER program database, which includes population-based data from 18 cancer registries and represents approximately $28 \%$ of the U.S population from 1975 to 2016 (18). SEER*Stat Software version 8.3.6 (https://seer.cancer.gov/seerstat/) (Information Management Service, Inc. Calverton, MD, USA) was used to generate the case listing. All procedures were performed in accordance with approved guidelines. This study was approved by the Ethics Committee of the First Affiliated Hospital of Xi'an Jiaotong University. The SEER data erases the identity information of patients, so there is no need for informed consent from the patients.

\section{Patient Cohort}

Female patients with pathologically confirmed breast cancer from 2010 to 2016 were enrolled in the study. Patients were included by following criteria: 1) primary breast cancer (ICD-0-3 histology codes: 8453/3, 8460/3, 8470/3, 8480/3, 8481/3, 8500/3, 8501/3, 8502/3, $8503 / 3,8504 / 3,8507 / 3,8510 / 3,8513 / 3,8520 / 3,8521 / 3,8523 / 3,8524 / 3,8540 / 3,8541 / 3,8560 / 3,8570 / 3,8574 / 3,8575 / 3) ; 2)$ TNM (Derived AJCC Stage Group, 7th ed (2010-2015), Derived SEER Cmb Stg Grp (2016+))stages T1N0M0; 3) aged 65 years or more; 4) ER positive and HER2 negative; 5)underwent breast conserving surgery.

After the preliminary selection, patients were excluded by following criteria: (1) unknown TNM stage; (2) the follow-up type of autopsy or death certificate; (3) unknown tumor size; (4) unknown PR status; (5) unknown laterality; (6)unknown RT status. The selecting procedure was shown in Figure 1.

A total of 52049 elderly patients with early breast cancer (EBC) were selected. The following demographic and clinicopathological variables were sex (female), tumor grade, age at diagnosis, race, primary site, year of diagnosis, tumor size, type of surgery, radiotherapy status, chemotherapy status, ER status, HER2 status, PR status, survival months, vital status, causes of death, marital status, and Derived AJCC TNM Stage Group, 7th ed (2010-2015) and Derived SEER Cmb TNM Stg Grp (2016+).

\section{End Points}

Patients were followed up until November 2018, and the median follow-up was 34 months (range 1-83 months). Our primary observation endpoint was OS, defined as the time from the date of diagnosis until death caused by any reasons. The secondary outcome measurements were BCSS and BCSD, defined as the date of diagnosis until death caused by breast cancer and the interval from the date of diagnosis to the date of death respectively.

\section{Statistics analysis}

The baseline characteristics of patients were described using summary statistics. One-to-one (1:1) propensity score matching (PSM) was used to balance baseline characteristics and potential prognostic confounders between the groups ${ }^{14}$. X-tile program was used to determine the cutoff points of optimal tumor size through comparing the survival between two sides of each tumor size and product a minimum p-value ${ }^{15}$. Fine and Gray multivariable regression model was performed to identify factors associated with risk of death from all causes, which aimed to reduce bias caused by informative censoring.

Furthermore, a competing risk analysis model was built to evaluate the impact of RT on BCSD after excluding the impact of other causespecific death (OCSD). SPSS version 23.0 (IBM Corporation, Armonk, NY, USA) and R software (version 3.6.2, R Foundation for Statistical Computing, Vienna, Austria. http://www. R-project. org/) were used for calculations. A two-sided p value $<0.05$ considered statistically significant. 


\section{Results}

\section{Baseline characteristics of patients}

Among the 52049 patients from our study cohort, a total of 35026 (67.3\%) patients received radiotherapy (RT group), while 17023 $(32.71 \%)$ had no radiotherapy (non-RT group). Of these patients with FBC, 31120 (59.8\%) of them aged between 65 years and 74 years,

$26113(50.2 \%)$ had tumors located in the left breast, and 26493 (50.9\%) had tumors located in the upper quadrant. In total, 45455 (87.3\%) patients were White, 25941 (49.8\%) of them got married. The majority of FBC were PR positive (89.5\%, 46570/52049), and tumor size ranging from $1 \mathrm{~mm}$ to $14 \mathrm{~mm}(73.8 \%, 38400 / 52049)$. In total, 22026 (42.3\%) cases were moderately differentiated (grade I), and 2061 (4.0\%) cases underwent chemotherapy. By comparing RT and non-RT groups, significant differences $(P<0.05)$ were found in age at diagnosis, year of diagnosis, marital status, primary site, histology, PR status, grade level, tumor size and chemotherapy status. After PSM, there was no significant difference between the groups except for the age which were grouped. The detailed methodological characteristics were shown in Table 1. 
Table 1

The clinical and pathological characteristics of patients before and after PSM.

\begin{tabular}{|c|c|c|c|c|c|c|c|c|}
\hline \multirow[t]{2}{*}{ Variable } & \multicolumn{4}{|l|}{ before PSM } & \multicolumn{4}{|l|}{ after PSM } \\
\hline & $\begin{array}{l}\text { Total } \\
\text { N (\%) }\end{array}$ & $\begin{array}{l}\text { non-RT } \\
\text { n (\%) }\end{array}$ & $\begin{array}{l}\text { RT } \\
\mathrm{n}(\%)\end{array}$ & $\begin{array}{l}\mathrm{P} \\
\text { value* }\end{array}$ & $\begin{array}{l}\text { Total } \\
\text { N (\%) }\end{array}$ & $\begin{array}{l}\text { non-RT } \\
\text { n (\%) }\end{array}$ & $\begin{array}{l}\text { RT } \\
\mathrm{n}(\%)\end{array}$ & $\begin{array}{l}P \\
\text { value* }\end{array}$ \\
\hline Total & 52049 & 17023(32.7) & $35026(67.3)$ & & 28068 & $14034(50.0)$ & $14034(50.0)$ & \\
\hline $\begin{array}{l}\text { Age at } \\
\text { diagnosis }\end{array}$ & & & & $<0.001$ & & & & 0.895 \\
\hline Mean(SD) & $73.80(6.70)$ & $77.37(7.25)$ & $72.07(5.66)$ & & $75.39(6.14)$ & $75.40(6.16)$ & $75.38(6.13)$ & \\
\hline $\begin{array}{l}\text { Age of } \\
\text { diagnosis }\end{array}$ & & & & $<0.001$ & & & & $<0.001$ \\
\hline $65-69$ & 17072(32.8) & 2685(15.8) & $14387(41.1)$ & & 5523(19.7) & 2684(19.1) & 2839(20.2) & \\
\hline $70-74$ & 14048(27.0) & $3967(23.3)$ & 10081(28.8) & & $7629(27.2)$ & 3966(28.3) & $3663(26.1)$ & \\
\hline $75-79$ & 10065(19.3) & $3696(21.7)$ & $6369(18.2)$ & & $7373(26.2)$ & $3590(25.6)$ & $3783(27.0)$ & \\
\hline$\geq 80$ & 10864(20.9) & $6675(39.2)$ & $4189(12.0)$ & & $7543(26.9)$ & $3794(27.0)$ & $3749(26.7)$ & \\
\hline $\begin{array}{l}\text { Year of } \\
\text { diagnosis }\end{array}$ & & & & $<0.001$ & & & & 0.514 \\
\hline 2010-2013 & 27499(52.8) & $8704(51.1)$ & 18795(53.7) & & $14668(52.3)$ & $7372(52.5)$ & $7296(52.0)$ & \\
\hline 2014-2016 & $24550(47.2)$ & $8319(48.9)$ & $16231(46.3)$ & & $13400(47.7)$ & $6662(47.5)$ & 6738(48.0) & \\
\hline Race & & & & 0.215 & & & & 0.736 \\
\hline White & 45455(87.3) & 14906(87.6) & $30549(87.2)$ & & 24465(87.2) & 12245(87.3) & $12220(87.1)$ & \\
\hline Black & $3240(6.2)$ & 1066(6.3) & $2174(6.2)$ & & $1810(6.4)$ & $900(6.4)$ & $910(6.5)$ & \\
\hline Others $^{a}$ & $3354(6.4)$ & 1051(6.2) & $2303(6.6)$ & & 1793(6.4) & $889(6.4)$ & $904(6.4)$ & \\
\hline Marital status & & & & $<0.001$ & & & & 0.835 \\
\hline Yes & $25941(49.8)$ & $7112(41.8)$ & 18829(53.8) & & 12951(46.1) & $6484(46.2)$ & $6467(46.1)$ & \\
\hline $\begin{array}{l}\text { No } \\
\text { b/Unknown }\end{array}$ & $26108(50.2)$ & $9911(58.2)$ & $16197(46.2)$ & & 15117(53.9) & $7550(53.8)$ & $7567(53.9)$ & \\
\hline Laterality & & & & 0.529 & & & & 0.107 \\
\hline Right & $25936(49.8)$ & $8447(49.6)$ & $17489(49.9)$ & & 13986(49.8) & $7087(50.5)$ & $6899(49.2)$ & \\
\hline Left & $26113(50.2)$ & $8576(50.4)$ & $17537(50.1)$ & & $14082(50.2)$ & $6947(49.5)$ & 7135(50.8) & \\
\hline Primary site & & & & $<0.001$ & & & & 0.987 \\
\hline $\begin{array}{l}\text { Upper } \\
\text { quadrant }\end{array}$ & $26493(50.9)$ & $8269(48.6)$ & $18224(48.6)$ & & 13951(49.7) & $6959(49.6)$ & 6992(49.8) & \\
\hline $\begin{array}{l}\text { Lower } \\
\text { quadrant }\end{array}$ & $7564(14.5)$ & $2437(14.3)$ & $5127(14.3)$ & & 3992(14.2) & 2023(14.4) & $1969(14.0)$ & \\
\hline Others $^{c}$ & 17992(34.6) & 6317(37.1) & 11675(37.1) & & 10125(36.1) & $5052(36.0)$ & $5073(36.1)$ & \\
\hline Histology & & & & $<0.001$ & & & & 0.633 \\
\hline $\begin{array}{l}\text { Duct } \\
\text { carcinoma }\end{array}$ & 41807(80.3) & 13667(80.3) & $28140(80.4)$ & & $22560(80.4)$ & $11279(80.4)$ & 11281(80.4) & \\
\hline $\begin{array}{l}\text { Lobular } \\
\text { carcinoma }\end{array}$ & $5048(9.7)$ & 1497(8.8) & 3551(10.1) & & 2611(9.3) & $1267(9.0)$ & $1344(9.6)$ & \\
\hline Others & 5194(10.0) & 1859(10.9) & $3335(9.5)$ & & 2897(10.3) & 1488(10.6) & $1409(10.0)$ & \\
\hline
\end{tabular}




\begin{tabular}{|c|c|c|c|c|c|c|c|c|}
\hline \multirow[t]{3}{*}{ Variable } & \multicolumn{4}{|l|}{ before PSM } & \multicolumn{4}{|l|}{ after PSM } \\
\hline & Total & non-RT & RT & $P$ & Total & non-RT & RT & P \\
\hline & $N(\%)$ & $\mathrm{n}(\%)$ & $\mathrm{n}(\%)$ & & $N(\%)$ & n (\%) & n (\%) & \\
\hline \multicolumn{4}{|l|}{ PR status } & 0.042 & & & & 0.788 \\
\hline Negative & $5479(10.5)$ & $1725(10.1)$ & $3754(10.7)$ & & 2898(10.3) & 1439(10.3) & 1459(10.4) & \\
\hline Positive & $46570(89.5)$ & 15298(89.9) & $31272(89.3)$ & & $25170(89.7)$ & 12595(89.7) & 12575(89.6) & \\
\hline \multicolumn{4}{|l|}{ Grade } & $<0.001$ & & & & 0.661 \\
\hline प & $22026(42.3)$ & 7634(44.8) & 14392(41.1) & & $12255(43.7)$ & 6123(43.6) & 6132(43.7) & \\
\hline ૫ & 25366(48.7) & $8025(47.1)$ & $17341(49.5)$ & & 13432(47.8) & 6692(47.7) & $6740(48.0)$ & \\
\hline $\mathbb{Z}-\mathbb{\square}$ & $4657(8.9)$ & 1364(8.0) & $3293(9.4)$ & & $2381(8.5)$ & 1219(8.7) & 1162(8.3) & \\
\hline \multicolumn{4}{|l|}{$\begin{array}{l}\text { Tumor } \\
\text { size }(\mathrm{mm})\end{array}$} & $<0.001$ & & & & 0.320 \\
\hline Mean (SD) & $10.81(4.66)$ & $10.67(4.73)$ & $10.88(4.62)$ & & $10.67(4.66)$ & $10.64(4.74)$ & $10.69(4.58)$ & \\
\hline \multicolumn{4}{|l|}{$\begin{array}{l}\text { Tumor } \\
\text { size }(\mathrm{mm})\end{array}$} & 0.089 & & & & 0.134 \\
\hline $1-14$ & $38400(73.8)$ & $12639(74.2)$ & $25761(73.5)$ & & $20975(74.7)$ & 10433(74.3) & $10542(75.1)$ & \\
\hline $15-20$ & $13649(26.2)$ & $4384(25.8)$ & $9265(26.5)$ & & 7093(25.3) & $3601(25.7)$ & $3492(24.9)$ & \\
\hline \multicolumn{4}{|l|}{ Chemotherapy } & $<0.001$ & & & & 0.496 \\
\hline No & 49988(96.0) & 16602(97.5) & 33386(95.3) & & 27222(97.0) & 13623(97.1) & 13599(96.9) & \\
\hline Yes & $2061(4.0)$ & $421(2.5)$ & $1640(4.7)$ & & $846(3.0)$ & $411(2.9)$ & $435(3.1)$ & \\
\hline \multicolumn{9}{|c|}{ Abbreviations: PSM, propensity score matching; RT, radiotherapy; SD, standard deviation; PR, progesterone receptor. } \\
\hline \multicolumn{9}{|c|}{ * $\mathrm{p}$-value was assessed using the Pearson's $\chi 2$ test. } \\
\hline \multicolumn{9}{|c|}{ a Including Non-Hispanic American Indian/Alaska Native, Non-Hispanic Asian or Pacific Islander, Non-Hispanic Unknown Race. } \\
\hline \multicolumn{9}{|c|}{ b Including divorced, separated, single (never married), and widowed. } \\
\hline
\end{tabular}

\section{X-tile Analysis Based On Os And Bcss}

To further explore and identify the optimal cutoff of tumor size which could affect the OS and BCSS rate for patients, X-tile analysis was used. Based on the cutoff value of $14 \mathrm{~mm}$ determined by X-tile analysis, $38400(73.8 \%)$ eligible patients were classified into small tumor group (tumor $\leq 14 \mathrm{~mm}$ in diameter), while 13649 (26.2\%) patients were classified into large tumor (tumor $\geq 15 \mathrm{~mm}$ in diameter) group. Small tumors were associated with better OS $(P<0.001)$ and BCSS $(P<0.001)$ in comparison with the large tumors, as shown in Figs. 2a and 2b, and better BCSS after the application of PSM (Additional file 1: Figure S1a). In addition, compared with large tumors, small tumors were also associated with better OS $(P<0.001)$ as well as BCSS $(P<0.001)$ for patients with RT, and better BCSS for patients without RT (Additional file 1: Figures S1b, 1c and 1d).

\section{Fine And Gray Multivariable Regression Model Analysis}

A total of 3846 deaths were included in the unmatched cohort, of which 11.93\% (459/3846) were BCSD, and 88.07\% (3387/3846) were OCSD. To further explore the independent predictive consequences of BCSD, fine and gray multivariable regression model analysis was performed (Table 2). Result showed that patients with large tumors had poorer BCSD (HR $=2.091,95 \% \mathrm{Cl}: 1.730-2.527, P<0.001)$ than 
those with small tumors. Patients in RT group ( $\mathrm{HR}=0.499,95 \% \mathrm{Cl}: 0.407-0.613, P<0.001)$ had lower BCSD than those in non-RT group. After PSM, patients with large tumors ( $\mathrm{HR}=2.024,95 \% \mathrm{Cl}: 1.578-2.583, P<0.001)$ still had worse BCSD than those with small tumors. In addition, after BCT, patients with highly differentiated grade I tumors, getting married, receiving RT or giving up chemotherapy tended to have significantly better BCSD than the corresponding subgroups $(P<0.05)$, regardless of before or after PSM. 
Table 2

Fine and Gray Multivariable Regression Model Analysis in patients aged 65 or older with T1NOM0 stage and ER+, HER2- breast cancer after BCT.

\begin{tabular}{|c|c|c|c|c|c|c|}
\hline \multirow[t]{2}{*}{ Variable } & \multicolumn{3}{|c|}{ before PSM } & \multicolumn{3}{|c|}{ after PSM } \\
\hline & HR & $95 \% \mathrm{Cl}$ & P value & HR & $95 \% \mathrm{Cl}$ & $P$ value \\
\hline Age at diagnosis & 1.045 & $1.03-1.059$ & $<0.001$ & 1.056 & $1.036-1.077$ & $<0.001$ \\
\hline \multicolumn{7}{|l|}{ Year of diagnosis } \\
\hline $2010-2013$ & 1 & & & 1 & & \\
\hline $2014-2016$ & 0.894 & $0.687-1.163$ & 0.400 & 0.963 & $0.698-1.330$ & 0.820 \\
\hline \multicolumn{7}{|l|}{ Race } \\
\hline White & 1 & & & 1 & & \\
\hline Black & 1.337 & $0.964-1.856$ & 0.082 & 1.517 & $1.015-2.268$ & 0.042 \\
\hline Others & 0.981 & $0.655-1.468$ & 0.920 & 1.008 & $0.598-1.702$ & 0.980 \\
\hline \multicolumn{7}{|l|}{ Marital status } \\
\hline Yes & 1 & & & 1 & & \\
\hline No/Unknown & 1.348 & $1.104-1.647$ & 0.003 & 1.341 & $1.037-1.734$ & 0.025 \\
\hline Primary site & & & & - & & \\
\hline Upper quadrant & 1 & & & - & & \\
\hline Lower quadrant & 1.074 & $0.823-1.403$ & 0.600 & - & & \\
\hline Others & 0.997 & $0.814-0.220$ & 0.970 & - & & \\
\hline Histology & & & & - & & \\
\hline Duct carcinoma & 1 & & & - & & \\
\hline Lobular carcinoma & 0.928 & $0.673-1.280$ & 0.650 & - & & \\
\hline Others & 0.738 & $0.525-1.038$ & 0.081 & - & & \\
\hline \multicolumn{7}{|l|}{ PR status } \\
\hline Negative & 1 & & & 1 & & \\
\hline Positive & 0.749 & $0.582-0.965$ & 0.025 & 0.755 & $0.547-1.043$ & 0.088 \\
\hline \multicolumn{7}{|l|}{ Grade } \\
\hline ૫ & 1 & & & 1 & & \\
\hline ૫ & 1.545 & $1.240-1.926$ & $<0.001$ & 1.297 & $0.991-1.697$ & 0.058 \\
\hline $\mathbb{Q}-\mathbb{\Delta}$ & 2.721 & $2.044-3.621$ & $<0.001$ & 2.170 & $1.491-3.159$ & $<0.001$ \\
\hline \multicolumn{7}{|l|}{ Tumor size(mm) } \\
\hline $1-14$ & 1 & & & 1 & & \\
\hline $15-20$ & 2.091 & $1.730-2.527$ & $<0.001$ & 2.024 & $1.587-2.583$ & $<0.001$ \\
\hline \multicolumn{7}{|l|}{ Chemotherapy } \\
\hline No & 1 & & & 1 & & \\
\hline Yes & 1.728 & $1.163-2.567$ & 0.007 & 1.797 & $1.006-3.209$ & 0.048 \\
\hline \multicolumn{7}{|l|}{ Radiotherapy } \\
\hline No & 1 & & & 1 & & \\
\hline
\end{tabular}




\begin{tabular}{|c|c|c|c|c|c|c|}
\hline \multirow[t]{2}{*}{ Variable } & \multicolumn{3}{|c|}{ before PSM } & \multicolumn{3}{|c|}{ after PSM } \\
\hline & HR & $95 \% \mathrm{Cl}$ & $P$ value & HR & $95 \% \mathrm{Cl}$ & $P$ value \\
\hline Yes & 0.499 & $0.407-0.613$ & $<0.001$ & 0.464 & $0.361-0.594$ & $<0.001$ \\
\hline
\end{tabular}

\section{Radiation Reduces The Risk Associated With Large Tumors}

To analyze the radiation-related survival in patients with small tumors and large tumors, a competitive risk model survival analysis was performed. A total of 38400 eligible patients were classified into small tumor group, of which 25761 (67.1\%) of them received RT (small tumor\& RT subgroup) and 12639 (32.9\%) did not (small tumor\& non-RT subgroup), while 13649 patients were classified into large tumor group, of which 9265 (67.9\%) of them received RT (large tumor \& RT subgroup) and 4384 (32.1\%) did not (large tumor \& non-RT subgroup). After competitive risk model survival analysis, as shown in Figs. $3 a$ and $3 b$, patients in small tumor \& non-RT subgroup had similar BCSD to those in the small tumor \& RT and large tumor\& RT subgroup, while patients in large tumor\& non-RT subgroup tended to have higher BCSD (Gray's test, $P<0.001$ ) and OCSD (Gray's test, $P<0.001$ ) than the other subgroups. In addition, patients in small tumor\& non-RT subgroup tended to have higher OCSD than small tumor\& RT and large tumor\& RT subgroups, whether before or after the application of PSM.

\section{Discussion}

At a median follow-up of 34 months, we demonstrate that tumor size of $14 \mathrm{~mm}$ was the optimal cutoff for predicting OS and BCSS for patients aged 65 years or older after BCT with clinical negative lymph nodes and ER-positive FBC. To our knowledge, this was the first and largest population-based study to assess the impact of tumor size with a cutoff as $14 \mathrm{~mm}$ on OS and BCSS using propensity score matching analysis, $\mathrm{X}$-tile analysis, survival variables, demographic and pathological factors.

OS, especially cancer specific survival (CSS) is an objective, reliable, and bias-free measurements for patients with FBC in long-term results. In our study, based on analysis of a large cohort of 52049 patients in SEER database from 2010 to 2016 and an integrated range of factors in competing risk model, small tumors $(\leq 14 \mathrm{~mm}$ ) were associated with better OS and BCSS for patients with radiotherapy as well as better BCSS for patients with no radiotherapy in comparison with large tumors ( $\geq 15 \mathrm{~mm})$ through X-title analysis. Such results indicated that whether with radiotherapy or not, large ER+, HER2-, and T1NOMO tumors was associated with poorer OS and BCSS and should be attended to for patients aged $\geq 65$ years after BCT, even if estimation and selection bias were possible.

To eliminate the estimation bias and further investigate the efficacy of tumor size on BCSS or other causes of death for FBC, Fine and Gray multivariable regression model analysis was performed. In our study, the patients with small tumors had better BCSD than those with large tumors. To minimize the selection bias resulting from baseline variables inherent in retrospective studies, PSM analysis was performed since PSM could eliminate a greater proportion of baseline differences between any two treatment groups than stratification or covariates adjustment. After PSM analysis, the tumor size $14 \mathrm{~mm}$ was still shown be the optimal cutoff for predicting BCSS. Patients with small tumors still had better BCSD than those with large tumors. These results suggested that small ER+, HER2-, and T1NOM0 tumors should be an independent indicator for patients aged $\geq 65$ years.

To further analyze and assess the efficacy of radiotherapy in patients with small or large tumors, a subgroup was performed. In our study, after BCT, patients in small tumors but no radiotherapy subgroup had similar BCSD to the radiotherapy subgroups with small tumors or large tumors, while patients in large tumors but no radiotherapy subgroup tended to have the highest BCSD, whether before or after the application of PSM. Such results showed, for patients aged $\geq 65$ years with ER+, HER2-, and T1N0M0 tumors after BCT, small tumors were associated with favorable prognosis, which suggested that radiotherapy could be abandoned with caution for that omitting radiotherapy did not decrease the BCSS rate of patients with a tumor size $\leq 14 \mathrm{~mm}$ significantly.

In addition, married patients with highly-differentiation level, and PR positive tumors tended to have better prognostic indicators with BCSD than the corresponding subgroups. These results consistent with the previous reports indicated that clinicopathological features, such as tumor differentiation level, TNM stage, PR status, marital status and income level are objective and reliable prognostic indicators in patients with breast carcinoma ${ }^{16-18}$. Moreover, patients receiving chemotherapy had worse BCSD than the corresponding subgroup. 
The underlying reason maybe that the local therapeutic effect of adjuvant chemotherapy on EBC patients cannot offset the systemic damage and long-term side effects ${ }^{19}$.

However,there are still some possible limitations in this study. Firstly, Selection bias may have occurred due to the nature of the retrospective analysis, for studies show that randomly assigned patients into different groups by treatment method are needed. Secondly, we were unable to avoid the possibility that the observed risk reductions might exclude the influence of potential confounders, such as family history, insurance coverage, patient anxiety, detailed regimens of endocrine therapy, frailty or co-morbid conditions known to be related to receipt of specific treatments, and so on. These data greatly impacted the clinical decisions and even breast cancer prognosis ${ }^{20-22}$. Thirdly, media follow-up in this study was merely 34 months. Longer follow-up times may be necessary for an accurate assessment of prognostic factors for patients with T1N0M0 FBC. Finally, and most importantly, there was a lack of information about local recurrence, adverse effects of normal tissue after radiotherapy. However, we believe that the findings of this study, which cover about $28 \%$ of the U.S. population of patients with cancer, are generalizable and will contribute to improved survival in elder patients with EBC after BCT.

\section{Conclusions}

Our study demonstrated that small tumors (tumor $\leq 14 \mathrm{~mm}$ in diameter) were associated with better OS, BCSD and OCSD, and radiotherapy might be omitted for patients aged 65 or older with T1NOMO stage, ER + and HER2- small breast tumors after BCT. Randomly controlled clinical trials are needed to provide a high-level evidence.

\section{Abbreviations}

\section{RT}

Radiotherapy

BCT

breast-conserving therapy

ER

estrogen receptor

PR

progesterone receptor

HER2

human epidermal growth factor receptor positive 2

SEER

Surveillance, Epidemiology, and End Results

\section{PSM}

propensity score matching

OS

overall survival

BCSD

breast cancer-specific death

OCSD

other cause-specific death

CSS

cancer specific survival

FBC

female breast cancer

EBC

early breast cancer

HR

hazard ratio

Cl

confidence interval 


\section{Declarations}

\section{Ethics approval and consent to participate}

This study was approved by the Ethics Committee of the First Affiliated Hospital of Xi'an Jiaotong University. The SEER data erases the identity information of patients, so there is no need for informed consent from the patients.

\section{Consent for publication}

Not applicable.

\section{Availability of data and materials}

We used data from an open database, the Surveillance, Epidemiology, and End Results (SEER) database (https://seer.cancer.gov), which are publicly available.

\section{Competing interests}

The authors declare that they have no competing interests.

\section{Funding}

This study was supported by the National Natural Science Foundation of China (NSFC 81502413 to C. Zhou and NSFC 81702633 to B. Wang) and Shaan'xi Provincial Natural Science Foundation of China (SNSFC 2019SF-145 to C. Zhou).

\section{Author's contributions}

Zejian Yang: data curation, statistical analysis, methodology, writing - original draft. Kunlong Li: statistical analysis, software, methodology. Bin Wang: Critical revision of manuscript, funding acquisition. Yu Yan: methodology, administrative. Chen Feng: formal analysis, critical revision of manuscript. Yu Ren: critical revision of manuscript. Du Meng: validation, critical revision of manuscript. Yijun Li: statistical analysis, technical and material support. Yifei Ma: statistical analysis, software. Pei Qiu: statistical analysis. Pingping Li: study concept and design, project administration, study supervision, analysis and interpretation of data. Can Zhou: conceptualization, study concept and design, acquisition of data, funding acquisition, study supervision.

\section{Acknowledgements}

Not applicable.

\section{Author details}

${ }^{1}$ Department of Breast Surgery, First Affiliated Hospital of Xi'an Jiaotong University, 277 Yanta Western Rd., Xi'an, 710061, Shaan'xi Province, China. ${ }^{2}$ Xi'an Jiaotong University Health Science Center, 76 Yanta Western Rd., Xi'an, 710061, Shaan'xi Province, China. ${ }^{3}$ Department of Translational Medicine, First Affiliated Hospital of Xi'an Jiaotong University, 277 Yanta Western Rd., Xi'an, 710061, Shaan'xi Province, China. ${ }^{4}$ Department of Oncology, Shaanxi Provincial Corps Hospital, Xi'an, 710061, Shaan'xi Province, China. ${ }^{5}$ Department of Radiotherapy, First Affiliated Hospital of Xi'an Jiaotong University, 277 Yanta Western Rd., Xi'an, 710061, Shaan'xi Province, China.

\section{References}

1. Darby S, McGale P, Correa C, et al. Effect of radiotherapy after breast-conserving surgery on 10-year recurrence and 15-year breast cancer death: meta-analysis of individual patient data for 10,801 women in 17 randomised trials. LANCET. 2011;378:1707-16.

2. Coates AS, Winer EP, Goldhirsch A, et al. Tailoring therapies-improving the management of early breast cancer: St Gallen International Expert Consensus on the Primary Therapy of Early Breast Cancer 2015. ANN ONCOL. 2015;26:1533-46.

3. Killander F, Karlsson P, Anderson H, et al. No breast cancer subgroup can be spared postoperative radiotherapy after breastconserving surgery. Fifteen-year results from the Swedish Breast Cancer Group randomised trial, SweBCG 91 RT. EUR J CANCER. 2016;67:57-65. 
4. Fisher ER, Anderson S, Tan-Chiu E, et al. Fifteen-year prognostic discriminants for invasive breast carcinoma: National Surgical Adjuvant Breast and Bowel Project Protocol-06. CANCER-AM CANCER SOC. 2001;91:1679-87.

5. Diab SG, Elledge RM, Clark GM. RESPONSE: Re: Tumor Characteristics and Clinical Outcome of Elderly Women With Breast Cancer. J Natl Cancer Inst. 2001;93:65-6.

6. Cabrera-Galeana P, Soto-Perez-de-Celis E, Reynoso-Noveron N, et al. Clinical characteristics and outcomes of older women with breast cancer in Mexico. J GERIATR ONCOL. 2018;9:620-5.

7. Matuschek C, Bolke E, Haussmann J, et al. The benefit of adjuvant radiotherapy after breast conserving surgery in older patients with low risk breast cancer- a meta-analysis of randomized trials. RADIAT ONCOL. 2017;12:60.

8. Hughes KS, Schnaper LA, Bellon JR, et al. Lumpectomy plus tamoxifen with or without irradiation in women age 70 years or older with early breast cancer: long-term follow-up of CALGB 9343. J CLIN ONCOL. 2013;31:2382-7.

9. Kunkler IH, Williams LJ, Jack WJ, et al. Breast-conserving surgery with or without irradiation in women aged 65 years or older with early breast cancer (PRIME II): a randomised controlled trial. LANCET ONCOL. 2015;16:266-73.

10. Kim YJ, Shin KH, Kim K. Omitting Adjuvant Radiotherapy for Hormone ReceptorPositive Early-Stage Breast Cancer in Old Age: A Propensity Score Matched SEER Analysis. CANCER RES TREAT. 2019;51:326-36.

11. Overgaard M, Hansen PS, Overgaard J, et al. Postoperative radiotherapy in high-risk premenopausal women with breast cancer who receive adjuvant chemotherapy. Danish Breast Cancer Cooperative Group 82b Trial. N Engl J Med. 1997;337:949-55.

12. McGale P, Taylor C, Correa C, et al. Effect of radiotherapy after mastectomy and axillary surgery on 10-year recurrence and 20-year breast cancer mortality: meta-analysis of individual patient data for 8135 women in 22 randomised trials. LANCET. 2014;383:212735.

13. Fyles AW, McCready DR, Manchul LA, et al. Tamoxifen with or without breast irradiation in women 50 years of age or older with early breast cancer. N Engl J Med. 2004;351:963-70.

14. Seeger JD, Williams PL, Walker AM. An application of propensity score matching using claims data. Pharmacoepidemiol Drug Saf. 2005; $14: 465-76$.

15. Camp RL, Dolled-Filhart M, Rimm DL. X-tile: a new bio-informatics tool for biomarker assessment and outcome-based cut-point optimization. CLIN CANCER RES. 2004;10:7252-9.

16. Gomez SL, O'Malley CD, Stroup A, et al. Longitudinal, population-based study of racial/ethnic differences in colorectal cancer survival: impact of neighborhood socioeconomic status, treatment and comorbidity. BMC CANCER. 2007;7:193.

17. Feller A, Schmidlin K, Bordoni A, et al. Socioeconomic and demographic disparities in breast cancer stage at presentation and survival: A Swiss population-based study. INT J CANCER. 2017;141:1529-39.

18. Waks AG, Winer EP. Breast Cancer Treatment: A Review JAMA. 2019;321:288-300.

19. Tao JJ, Visvanathan K, Wolff AC. Long term side effects of adjuvant chemotherapy in patients with early breast cancer. BREAST. 2015;24(Suppl 2):149-53.

20. Jagsi R, Hawley ST, Griffith KA, et al. Contralateral Prophylactic Mastectomy Decisions in a Population-Based Sample of Patients With Early-Stage Breast Cancer. JAMA SURG. 2017;152:274-82.

21. Boughey JC, Hoskin TL, Degnim AC, et al. Contralateral prophylactic mastectomy is associated with a survival advantage in high-risk women with a personal history of breast cancer. ANN SURG ONCOL. 2010;17:2702-9.

22. Brennan ME, McKessar M, Snook K, et al. Impact of selective use of breast MRI on surgical decision-making in women with newly diagnosed operable breast cancer. BREAST. 2017;32:135-43.

\section{Figures}




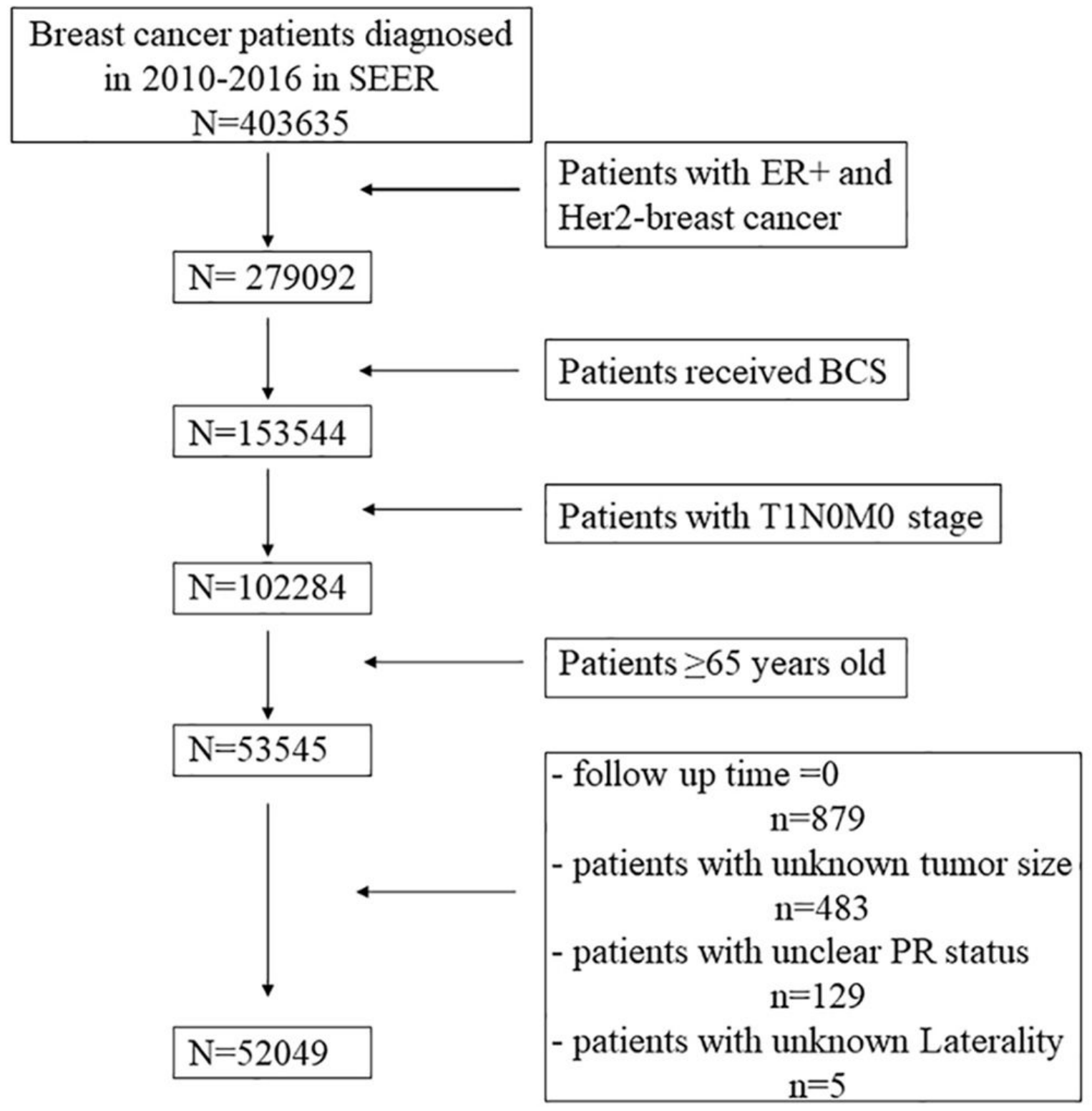

Figure 1

Eligibility, inclusion, and exclusion criteria of study population.

a
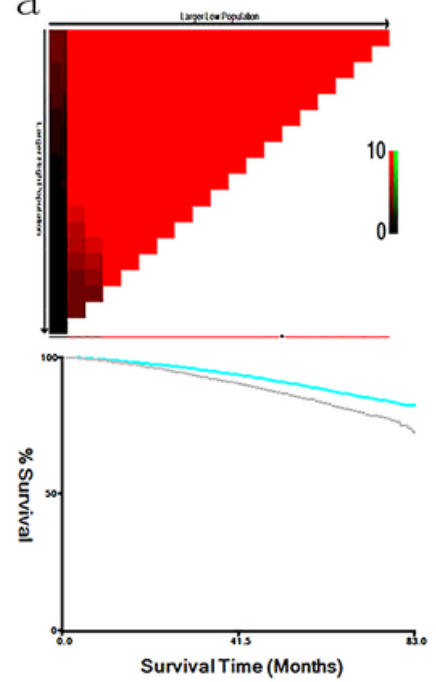
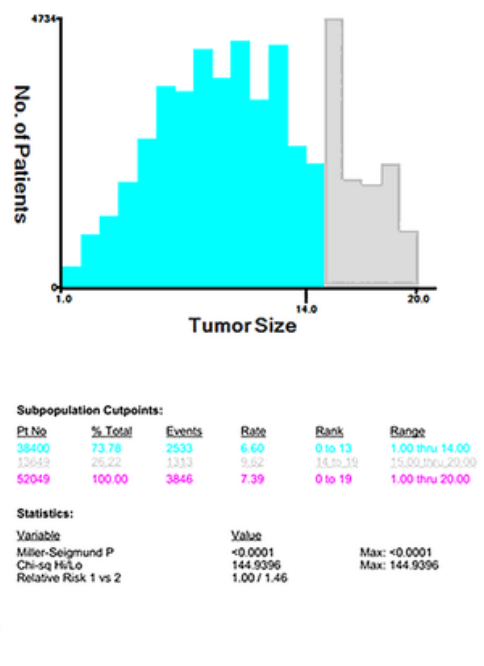

$\mathrm{b}$
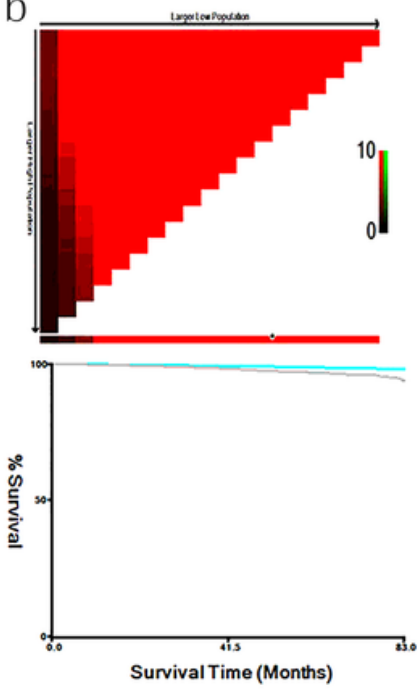


\section{Figure 2}

X-tile analysis of survival data from the SEER registry. The optimal cutoff points of tumor size were obtained based on overall survival (OS) (a) and breast cancer specific survival (BCSS) (b) of the whole population, before the application of propensity score matching (PSM). Each graph contains the X-tile plot, a histogram, the K-M curve, and the data related to optimal cut-point.

a

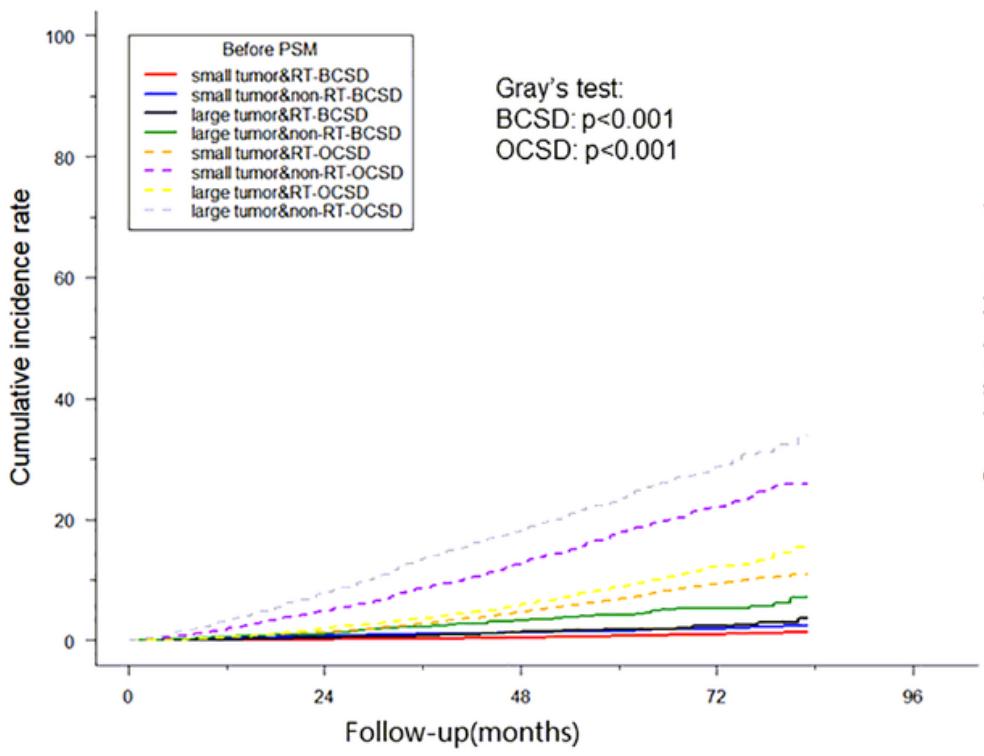

b

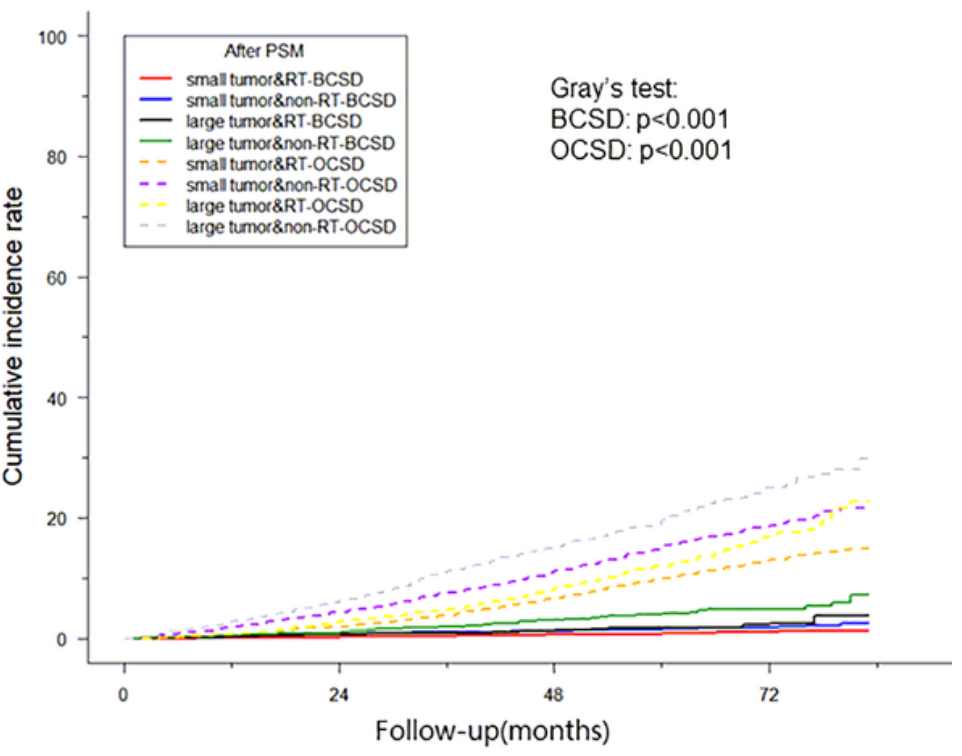

Figure 3

(a) Cumulative incidence of breast cancer specific death (BCSD) and other cause specific death (OCSD) in subgroups associated with tumor size and radiotherapy before PSM. (b) Cumulative incidence of BCSD and OCSD in subgroups associated with tumor size and radiotherapy after PSM.

\section{Supplementary Files}

This is a list of supplementary files associated with this preprint. Click to download.

- Additionalfile.docx 